\title{
Power-Delay Tradeoffs in Green Wireless Access Networks
}

\author{
Farah Moety*, Samer Lahoud*, Bernard Cousin*, Kinda Khawam ${ }^{\dagger}$ \\ *University of Rennes I - IRISA, France \\ †University of Versailles - PRISM, France
}

\begin{abstract}
Targeting energy efficiency while meeting user Quality of Service $(\mathrm{QoS})$ is one of the most challenging problems in future wireless networks. Since base stations (BSs) consume a high percentage of the total energy used in a wireless access network, saving power at the BS level is a major concern in green networks.

In this paper, we propose an optimization model based on finding a tradeoff between reducing the number of active radio cells and increasing the transmit power of BSs to better serve all users in the system. The main contribution of the paper is the formulation of a multiobjective optimization problem that jointly minimizes the network power consumption and the sum of data unit transmission delays of all users in the network. Our proposed model is solved using an exhaustive search algorithm to obtain the optimal solution. Solving the optimization problem at hand is very challenging due to the exhaustive search high computational complexity. Therefore, we run simulations in a small network to give insights into the optimal solution. Specifically, we study different cases by tuning the weights of the power and delay costs. This is a distinctive and important feature of our model allowing it to reflect various decision preferences. Regarding these preferences and under various users spatial distribution, results show that our solution allows to select the optimal network configuration in terms of power consumption while guaranteeing minimal delay for all users in the network.
\end{abstract}

\section{INTRODUCTION}

Global energy consumption becomes a major issue, because of its significant environmental footprint and eventual exhaustion of traditional energy resources. Current estimates indicate that Information and Communication Technology (ICT) is responsible for a fraction of the world energy consumption ranging between $2 \%$ and $10 \%$ [3]. As a branch of the sector, wireless access networks are responsible for $0.5 \%$ of this consumption. This situation, coupled with an increasing energy cost, has generated an urgent need to address the development of more energy efficient green communications. From the perspective of mobile operators, reducing energy consumption is not only an ecological concern, it is also an economic incentive. Precisely, the cost for running a network is largely affected by the energy bill and significant savings in capital expenditure and operational expenditure can be realized through reduced energy needs [4]. Moreover, mobile data traffic has grown at a compound annual growth rate of 131 $\%$ between 2008 and 2013, and will exceed two exabyte per month in 2013 [5]; this incredible increase will necessitate continually higher energy consumption. Therefore, the faced challenge is how to design future wireless networks to be more energy efficient while accommodating the extra traffic and satisfying Quality of Service (QoS) requirements of the users in the network.

Currently over $80 \%$ of the power in mobile telecommunications is consumed in the radio access network, more specifically at the base stations (BSs) level [4]. Hence, most of studies on energy efficiency in wireless network focus on the radio access. In the literature, various techniques are proposed for improving energy efficiency in wireless access networks such as designing energy efficient hardware, decreasing the cell size [6], adapting power consumption to traffic load [7] and devising intelligent network deployment strategies using small, low power, femtocells and relays [4][8]-[10]. However satisfying user QoS has not been considered as a constraint in these papers. A notable exception are the works in [11] and [12]. In [11] authors proposed an optimization approach based on an Integer Linear Programming (ILP) model that minimizes power consumption in wireless access networks while ensuring coverage of active users and enough capacity for guaranteeing QoS. Results showed possible saving through optimized management of on/off state and transmission power of access stations according to traffic estimates in different hours of the day or days of the week. In [12], authors formulated a cost minimization problem that allows for a flexible tradeoff between flow-level performance and energy consumption. Results showed that their proposed energyefficient user association and BS operation algorithms can dramatically reduce the total energy consumption, depending on the traffic arrival rate, spatial distribution and density.

In this paper, we tackle the problem of power saving while minimizing the user delay in wireless access networks by finding a tradeoff between reducing the number of active BSs and increasing the transmit power of those that remain active to better serve all users in the network. Thus, we formulate a multiobjective optimization problem that jointly minimizes the power consumption of the network and the sum of data unit transmission delays of all users in the network. Compared to prior work in the state of the art taking into consideration power saving and QoS: the optimization approach proposed in [11] does not support the feature of tuning the weights associated to the power and QoS costs; and [12] uses a M/GI/1 queue for the delay model, which is a pessimistic bound compared to the realistic delay model we use in our paper. Moreover, [12] study only the case where BSs switch between on and off modes without adjusting their transmit power.

Switching BSs into sleep/active mode must be coupled with 
user association: when an active BS switches to sleep mode or to a different transmit power level, users may need to change their associations. This coupling makes the problem more challenging. In our formulation, we consider the case of a Wireless Local Area Network (WLAN) using IEEE 802.11g technology. The design variable in this problem is to decide what follows:

- The running mode (sleep/active) of the network BSs and for active BSs, the corresponding transmit power level.

- The users association in the network.

The key contributions of our work are as follows:

- We formulate the problem of power-delay minimization in wireless access networks, going beyond the prior work in the literature which has focused either on minimizing energy without taking into consideration the QoS (i.e., delay) [4][6]-[10] or on delay analysis without taking into account energy minimization [1][2]. Hence, the novelty in our approach is that it does not only strive to save energy by reducing power consumption but also takes into consideration the user satisfaction by maximizing its perceived rate.

- Our problem formulation is multiobjective. Thus, it allows investigating the power-delay tradeoffs by tuning the weights associated to the network total cost components, namely power and delay. This is an important feature of our model allowing it to reflect various preferences (i.e., minimizing power, minimizing delay or jointly minimizing power and delay).

- Our optimization problem is solved using an exhaustive search algorithm to obtain the optimal network configuration. However, the exhaustive search is very computational intensive for large scenarios and thereby solving the optimization problem is very challenging. Therefore, to give insights into the optimal solution we provide extensive simulations for a small network where different scenarios are considered regarding the spatial users distribution.

- The delay model provided in this paper is a unique feature of our work, it is the realistic model used in WiFi [13].

The rest of the paper is organized as follows. In Section II, we formally describe the system model considering a 802.11 WLAN. In Section III we present our proposed optimization approach. In Section IV we provide extensive simulation results. Conclusions and perspectives are given in Section V.

\section{NEWORK MODEL}

We consider the energy consumption of IEEE $802.11 \mathrm{~g}$ WLAN in infrastructure mode. Similar considerations can be done for other wireless technologies, including 3G, WiMAX, or LTE systems. We refer in the following to a BS by an Access Point (AP) as we consider the case of WLANs. As the downlink traffic on mobile networks is still today several orders higher than the uplink traffic, we only consider the downlink traffic (e.g., accessing web data) sent from AP to user devices. We assume that the network is in a static state where users are stationary. In other words, we take a snapshot of a dynamic system and optimize its current state. Furthermore, we assume that the network is in a saturation state, which means that we treat a worst case scenario where every user has persistent traffic. Moreover, the APs operate in two modes: sleep mode and active mode. In the active mode, an AP is able to transmit at different power levels leading to different coverage levels. We denote by $N_{a p}$ and $N_{l}$ the number of APs in the network and the number of transmit power levels respectively. The indexes $i \in I=1, \ldots, N_{a p}$, and $j \in J$ $=0,1, \ldots, N_{l}$, are used throughout the paper to designate a given AP and its transmit power level respectively. We term by $k \in K=1, \ldots, N_{u}$, the index of a given user device where $N_{u}$ is the number of user devices in the network.

\section{A. Power Consumption Model}

Adopting the proposed model in [4], the power consumption of an AP is modeled as a linear function of average transmit power per site as below:

$$
p_{i, j}=L \cdot\left(a \pi_{j}+b\right)
$$

where $p_{i, j}$ and $\pi_{j}$ denote the average consumed power per AP $i$ and the transmit power at level $j$ respectively. The coefficient $a$ accounts for the power consumption that scales with the transmit power due to radio frequency amplifier and feeder losses while $b$ models the power consumed independently of the transmit power due to signal processing and site cooling. $\mathrm{L}$ reflects the activity level of the APs. As we assume that the network is in a saturation state, $\mathrm{L}$ is equal to one, e.g., each active AP has at least one mobile requesting data with all resources allocated. We consider that the average power consumed by an AP in sleep mode is negligible, hence $p_{i, 0}=$ $0 \forall i=1, \ldots, N_{a p}$. Whereas, in the active mode the average power consumed by an AP depends on its transmit power level.

\section{B. Delay Model}

Considering only the downlink traffic and neglecting the 802.11 waiting times (i.e., $\mathrm{DIFS}^{1}, \mathrm{SIFS}^{2}$ ), the CSMA/CA ${ }^{3}$ access scheme leads to a fair rate sharing of the radio channel among user devices [13]. Thus, all user devices have the same throughput. This throughput is less than the minimum peak rate that a single user can perceive when alone in the cell [1]. The peak rate of each user depends on his radio conditions and on the power level of the transmitting AP he is associated to. We denote by $\chi_{i, k, j}$ the peak rate perceived by user $k$ from AP $i$ transmitting at level $j$. Therefore, when user $k$ is associated to AP $i$ transmitting at level $j$, his perceived data rate is given by [2]:

$$
R_{i, k, j}=\frac{1}{\frac{1}{\chi_{i, k, j}}+\sum_{k^{\prime}=1, k^{\prime} \neq k}^{N_{u}} \frac{\theta_{i, k^{\prime}}}{\chi_{i, k^{\prime}, j}}}
$$

\footnotetext{
${ }^{1}$ DIFS: Distributed Coordination Function Interframe Space ${ }^{2}$ SIFS: Short Interframe Space

${ }^{3}$ CSMA/CA: Carrier Sense Multiple Access with Collision Avoidance
} 
where,

$$
\theta_{i, k^{\prime}}= \begin{cases}1 & \text { if user } k^{\prime} \text { is associated to } \operatorname{AP} i \\ 0 & \text { otherwise }\end{cases}
$$

We denote by $T_{i, k, j}$ the amount of time necessary to send a data unit to user $k$ from $\mathrm{AP} i$ transmitting at level $j$. In fact, the delay needed to transmit a bit for a given user is the inverse of the throughput perceived by this user. Therefore, we deduce from Equation 2 the following:

$$
T_{i, k, j}=1 / R_{i, k, j}=\frac{1}{\chi_{i, k, j}}+\sum_{k^{\prime}=1, k^{\prime} \neq k}^{N_{u}} \frac{\theta_{i, k^{\prime}}}{\chi_{i, k^{\prime}, j}} .
$$

Note that this model can be easily adapted to other wireless technologies such as 2G, WiMAX, or LTE systems [2].

\section{OPTIMIZATION PROBLEM}

\section{A. Problem Formulation}

Our Approach can be formulated as an optimization problem $(\mathcal{P})$ that consists in minimizing the power consumption of the network and the sum of the data unit transmission delays of all users in the network. These two minimization objectives are conflictual since the bit transmission delay decreases as the transmit power of the AP increases. Thus, a joint optimization of power and delay costs enables to tune the impact of each cost. Our problem $(\mathcal{P})$ uses two sets of decision variables:

- $\theta_{i, k}$ represents user association as defined in section II-B.

- $\lambda_{i, j}$ indicates the transmit power level of an AP and is defined as follows:

$$
\lambda_{i, j}= \begin{cases}1 & \text { if AP } i \text { is transmitting at level } j \\ 0 & \text { otherwise. }\end{cases}
$$

We define the power cost and the delay cost as follows:

The power cost, denoted by $C_{p}\left(\lambda_{i, j}\right)$, is defined as the total power consumption of active APs in the network. The power consumption of an active AP is given in Equation 1. Thus $C_{p}\left(\lambda_{i, j}\right)$ is expressed as follows:

$$
\begin{array}{r}
C_{p}\left(\lambda_{i, j}\right)=\sum_{i=1}^{N_{a p}} p_{i}\left(\lambda_{i, j}\right)=\sum_{i=1}^{N_{a p}} \sum_{j=0}^{N_{l}} p_{i, j} \cdot \lambda_{i, j} \\
=\sum_{i=1}^{N_{a p}} \sum_{j=0}^{N_{l}}\left(a \pi_{j}+b\right) \cdot \lambda_{i, j}
\end{array}
$$

The delay cost, denoted by $C_{d}\left(\theta_{i, k}, \lambda_{i, j}\right)$, is defined as the sum of data unit transmission delays of all users. The data unit transmission delay of a user is given in Equation 3. Thus $C_{d}\left(\theta_{i, k}, \lambda_{i, j}\right)$ is expressed as follows:

$$
\begin{array}{r}
C_{d}\left(\theta_{i, k}, \lambda_{i, j}\right)=\sum_{i=1}^{N_{a p}} \sum_{k=1}^{N_{u}} T_{k}\left(\lambda_{i, j}\right) \cdot \theta_{i, k} \\
=\sum_{i=1}^{N_{a p}} \sum_{k=1}^{N_{u}} \sum_{j=0}^{N_{l}} T_{i, k, j} \cdot \lambda_{i, j} \cdot \theta_{i, k}
\end{array}
$$

\begin{tabular}{|l|l|}
\hline Notation & Definition \\
\hline$N_{a p}$ & The number of APs \\
\hline$N_{l}$ & The number of transmit power levels \\
\hline$N_{u}$ & The number of user devices \\
\hline$p_{i, j}$ & $\begin{array}{l}\text { The average consumed power per AP } i \\
\text { transmitting at power level } j\end{array}$ \\
\hline$\pi_{j}$ & The transmit power at level $j$ \\
\hline$\chi_{i, k, j}$ & $\begin{array}{l}\text { The peak rate perceived by user } k \\
\text { from AP } i \text { transmitting at level } j\end{array}$ \\
\hline$T_{i, k, j}$ & $\begin{array}{l}\text { The amount of time necessary to send a data unit to user } k \\
\text { from AP } i \text { transmitting at level } j\end{array}$ \\
\hline$\theta_{i, k}$ & $\begin{array}{l}\text { A binary variable that indicates whether user } k \\
\text { is connected to AP } i\end{array}$ \\
\hline$\lambda_{i, j}$ & $\begin{array}{l}\text { A binary variable that indicates whether an AP } i \\
\text { transmits at power level } j\end{array}$ \\
\hline
\end{tabular}

Table I

NOTATION SUMMARY

Consequently, the network total cost, denoted by $C_{t}\left(\theta_{i, k}, \lambda_{i, j}\right)$, is defined as the weighted sum of power and delay costs and is given by the following:

$$
C_{t}\left(\theta_{i, k}, \lambda_{i, j}\right)=\alpha C_{p}\left(\lambda_{i, j}\right)+\beta \beta^{\prime} C_{d}\left(\theta_{i, k}, \lambda_{i, j}\right)
$$

$\beta^{\prime}$ is a normalization factor and $\alpha$ and $\beta$ are the weighting factors that tune the tradeoff between the two components of the network total cost. $\alpha$ and $\beta \in[0,1]$ : in particular when $\alpha$ equals 1 and $\beta$ equals 0 , we only focus on power saving, and as $\alpha$ decreases and $\beta$ increases more emphasis is given to the delay cost.

Our problem $(\mathcal{P})$ consists in finding an optimal set of active APs transmitting at a specific power level and an optimal user association that minimize the network total cost $C_{t}\left(\theta_{i, k}, \lambda_{i, j}\right)$. Therefore $(\mathcal{P})$ is given by:

$\operatorname{Min} C_{t}\left(\theta_{i, k}, \lambda_{i, j}\right)=\alpha \sum_{i=1}^{N_{a p}} p_{i}\left(\lambda_{i, j}\right)+\beta \beta^{\prime} \sum_{i=1}^{N_{a p}} \sum_{k=1}^{N_{u}} T_{k}\left(\lambda_{i, j}\right) \cdot \theta_{i, k}$

Subject to:

$$
\begin{aligned}
& \sum_{i=1}^{N_{a p}} \theta_{i, k}=1, \quad \forall k \in K=1, . ., N_{u} \\
& \sum_{j=0}^{N_{l}} \lambda_{i, j}=1, \quad \forall i \in I=1, . ., N_{a p}
\end{aligned}
$$

The objective function (7) provides the total cost of the network in terms of power and delay. Constraints (8) ensure that a given user is connected to only one AP. Constraints (9) state that every AP transmits only at one power level. The main notations used in the present paper are reported in Table I.

\section{B. Optimal Solution}

Problem $(\mathcal{P})$ is a non-linear optimization that consists in minimizing the objective function 7 subject to the constraints 8 and 9 . Solving problem $(\mathcal{P})$ gives:

- The running mode of each AP and its corresponding transmit power. This is designated by $\lambda_{i, j}$ for each $i \in I$ and $j \in J$.

- The users association designated by $\theta_{i, k}$ for each $i \in I$ and $k \in K$. 

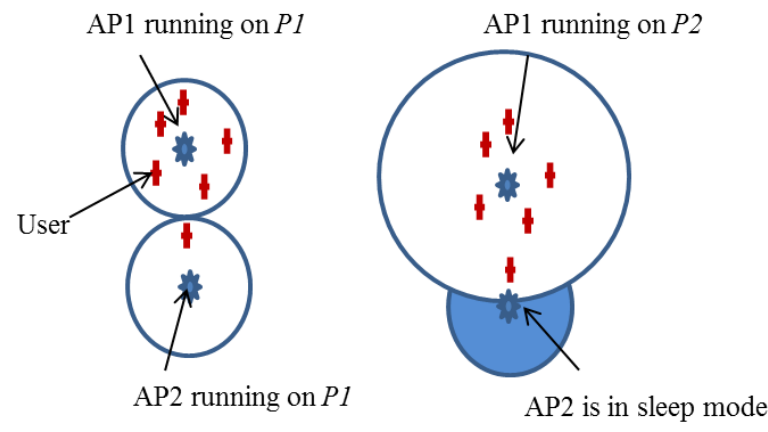

Figure 1. Examples of the APs state in the network.

In this paper, we propose to compute the optimal solution of $(\mathcal{P})$ using an exhaustive search algorithm. Such an algorithm explores all the possible solutions to compute the minimum of the objective function. We note that the time complexity of this exhaustive search algorithm is function of the number of user devices, the number of APs and the corresponding number of transmit power levels. Indeed, the objective function should be evaluated for each value of $\theta_{i, k}$ and $\lambda_{i, j}$. As $\theta_{i, k}=0$ or 1 for each $i=1, \ldots, N_{a p}$ and $k=1, \ldots, N_{u}$, then the cardinality of the set of values corresponding to $\theta_{i, k}$ is $2^{N_{a p} \cdot N_{u}}$. Same applies for $\lambda_{i, j}$, the cardinality of the set of values corresponding to $\lambda_{i, j}$ is $2^{N_{a p} \cdot\left(N_{l}+1\right)}$. Hence the time complexity for computing the minimum value of the objective function over these sets is in $\mathcal{O}\left(2^{N_{a p} \cdot N_{u}} \cdot 2^{N_{a p} \cdot\left(N_{l}+1\right)}\right)=\mathcal{O}\left(2^{N_{a p} \cdot\left(N_{u}+N_{l}+1\right)}\right)$. Thus, the exhaustive search is very computational intensive, and rapidly becomes intractable for large scenarios.

\section{Performance Evaluation}

\section{A. Evaluation Methodology}

In order to assess the tradeoff between power and delay in WLANs, we have implemented an exhaustive search algorithm in MATLAB to compute the optimal solution of the problem. As the exhaustive search has high computational complexity for large scenarios, we consider a small network consisting of two cells covered by IEEE $802.11 \mathrm{~g}$ technology and six users. The location of these users in the network are generated randomly following a uniform distribution.

Without loss of generality, we consider that in active mode, WLAN APs denoted by AP1 and AP2 are able to transmit for instance at two power levels and consequently they will have two coverage areas. We denote by:

- $S$ : the sleep mode state

- P1: the first power level state

- P2: the second power level state

In Figure 1, we give two examples of the APs state in the network. In the first example, the two APs run on the first power level state $P 1$, while in the second one AP1 runs on the second power level state $P 2$ and AP2 is in sleep mode.

The input parameters of the power consumption model in Equation 1 are given below [10]:

- $a=3.2, b=10.2$

- $\pi_{1}=0.01 \mathrm{~W}$ and $\pi_{2}=0.04 \mathrm{~W}$ the transmit power when the AP is running on $P 1$ and $P 2$ respectively.

\begin{tabular}{|c|c|}
\hline Parameter & Value \\
\hline$N_{a p}$ & 2 \\
\hline$N_{l}$ & 2 \\
\hline$N_{u}$ & 6 \\
\hline$a$ & 3.2 \\
\hline$b$ & 10.2 \\
\hline$\pi_{1}$ & 0.01 \\
\hline$\pi_{2}$ & 0.04 \\
\hline
\end{tabular}

Consequently, the average consumed power per AP $i$ at the first and the second power levels and are given respectively by $p_{i, 1}=10.232 \mathrm{~W}$ and $p_{i, 2}=10.328 \mathrm{~W}(i=1,2)$. In addition, the coverage radius for the first and the second power levels are respectively $70 \mathrm{~m}$ and $140 \mathrm{~m}$. As mentioned earlier, we assume that $p_{i, 0}=0(i=1,2)$ when the APs are in sleep mode. In the power consumption model of an AP, the parameter $b$ is large compared to the parameter $a$. Thus increasing transmit power will not contribute in a significant amount to the total power consumption since the transmit power is very low compared to the total power consumption of an active AP. Therefore, in a WLAN network, power can be saved more efficiently by switching an active AP to sleep mode and by increasing the transmit power of one of its neighbor AP. Table II depicts a summary of the main parameters considered in our simulation.

For the delay model, the peak rate input parameter $\chi_{i, k, j}$ for each user $k$ is obtained using Network Simulator (NS2). In particular, we implement in NS2 a benchmark scenario consisting of one IEEE 8021.11g AP working on $2.462 \mathrm{GHz}$ (channel 11) and a single user at different positions. This user is receiving from the access point a Constant Bit Rate (CBR) traffic with a packet size of 1000 bytes and an interarrival time of $0.4 \mathrm{~ms}$ corresponding to a rate of $20 \mathrm{Mb} / \mathrm{s}$. This leads to saturation state of the network according to the assumption presented in section II. In these conditions, the throughput experienced by our single user is the maximum achievable throughput (peak rate) for the current signal-tonoise ratio (SNR). Moreover, changes in transmit power have an influence on the radio channel conditions. Therefore, we run this scenario for each transmit power level $\left(\pi_{1}=0.01 \mathrm{~W}\right.$ and respectively $\pi_{2}=0.04 \mathrm{~W}$ ) of the AP to obtain respectively $\chi_{i, k, 1}$ and $\chi_{i, k, 2}$ for the corresponding user. Additionally, to characterize the WLAN radio environment, we use the Free Space propagation model.

Toward studying the tradeoff between minimizing the power consumption of the network and minimizing the delay of all users in the network; we tune the values of the weights $\alpha$ and $\beta$ associated to power and delay costs respectively, and investigate the obtained solutions. We consider three settings: the two weights are equal, $\beta$ is very large compared to $\alpha$, and $\alpha$ is very large compared to $\beta$. Practically the first setting matches the case where the power and delay components of the network total cost are equally important. The second setting matches the case where more preference is given to the delay cost representing the user QoS. On the opposite, the third setting matches the case where more importance is given to 
power saving.

For each case we run four scenarios regarding spatial users distribution in the two cells:

- Scenario I: all users are in the coverage area of AP2 running on $P 1$.

- Scenario II: $17 \%$ of users are in the coverage area of AP1 running on $P 1$.

- Scenario III: $33 \%$ of users are in the coverage area of AP1 running on $P 1$.

- Scenario IV: $50 \%$ of users are in the coverage area of AP1 running on $P 1$.

Table III illustrates the different scenarios studied in the simulation for the three considered cases.

Table III

SUMMARY OF THE SCENARIOS CONSIDERED IN EACH CASE IN THE SIMULATION

\begin{tabular}{|c|c|c|c|}
\hline & $\begin{array}{c}\text { Case 1: } \\
\alpha=\beta=1\end{array}$ & $\begin{array}{c}\text { Case 2: } \\
\alpha=0.01 ; \beta=0.99\end{array}$ & $\begin{array}{c}\text { Case 3: } \\
\alpha=0.99 ; \beta=0.01\end{array}$ \\
\hline Scenario I & \multicolumn{3}{|c|}{ All users are covered by AP2 running on $P 1$} \\
\hline Scenario II & \multicolumn{3}{|c|}{$17 \%$ of users are covered by AP1 running on $P 1$} \\
\hline Scenario III & \multicolumn{3}{|c|}{$33 \%$ of users are covered by AP1 running on $P 1$} \\
\hline Scenario IV & \multicolumn{3}{|c|}{$50 \%$ of users are covered by AP1 running on $P 1$} \\
\hline
\end{tabular}

We run each scenario 100 times (100 snapshots), and for each instance: we generate the positions of users, then using the benchmark implemented in NS2 we map each user position with the corresponding peak rate $\chi_{i, k, j}$ for each user $k$. Afterwards, as the generation of input data is performed, our problem $(\mathcal{P})$ is solved. For each optimal solution, we note the state of the APs and the network total cost defined in Equation 6 as the weighted sum of power and delay. The different snapshots of the network with different users distributions enables to capture the different states of a dynamic network over time. Accordingly, the number of occurrence for a state of a given AP reflects the time spent by the AP in this state. Therefore, for each scenario, we calculate the occurrence of each state of the APs in the 100 snapshots. This will help us decide on which state to run the APs in the network for the considered case. Moreover, we calculate the $95 \%$ Confidence Interval (CI) of the network total cost for the different considered scenarios. Note that we choose the normalization factor $\beta^{\prime}$ in such a way to scale the two components of the network total cost.

\section{B. Simulation Results}

Starting with the first case where $\alpha=\beta=1$, we give the same emphasis for the power and delay components of the network total cost. Table IV shows the occurrence of each

\begin{tabular}{|c|c|c|c|c|c|c|c|}
\hline \multicolumn{4}{|c|}{ (a) Scenario I } & \multicolumn{4}{|c|}{ (b) Scenario II } \\
\hline & Pl & $P 2$ & $S$ & & $P 1$ & $P 2$ & $S$ \\
\hline AP1 & 0 & 0 & $100 \%$ & AP1 & $4 \%$ & $90 \%$ & $6 \%$ \\
\hline AP2 & 0 & $100 \%$ & 0 & AP2 & 0 & $100 \%$ & 0 \\
\hline
\end{tabular}

(c) Scenarios III and IV

\begin{tabular}{c|c|c|c} 
& $P 1$ & $P 2$ & $S$ \\
\hline AP1 & 0 & $100 \%$ & 0 \\
\hline AP2 & 0 & $100 \%$ & 0
\end{tabular}

Table IV

THE APS STATE FOR THE DIFFERENT SCENARIOS FOR CASE $1(\alpha=\beta=1)$.

\begin{tabular}{|c|c|c|c|c|c|c|c|}
\hline \multicolumn{4}{|c|}{ (a) Scenario I } & \multicolumn{4}{|c|}{ (b) Scenarios II, III and IV } \\
\hline & P1 & $P 2$ & $S$ & & $P 1$ & $P 2$ & $S$ \\
\hline AP1 & 0 & $95 \%$ & $5 \%$ & AP1 & 0 & $100 \%$ & 0 \\
\hline AP2 & 0 & $100 \%$ & 0 & AP2 & 0 & $100 \%$ & 0 \\
\hline
\end{tabular}

Table V

THE APS STATE FOR THE DIFFERENT SCENARIOS FOR CASE $2(\alpha=0.01$ AND $\beta=0.99$ ).

state of the APs for the considered scenarios for the first case. As expected, in scenario I where all users are in the coverage area of the AP2 (Table IV(a)), AP2 runs always on the second power level $P 2$ and AP1 is in sleep mode. In scenario II (Table IV(b)), for $90 \%$ of the snapshots AP1 runs on P2, and AP2 runs always on $P 2$. For the remaining scenarios, the two APs run on $P 2$ as shown in Table IV(c). For the case where delay and power costs are equally important, power can be saved in scenarios when there is no user in a cell by putting the corresponding AP into sleep mode. While for the remaining scenarios, we put the two APs on $P 2$ due to the fact that we are not only minimizing power consumption but also the transmission delay.

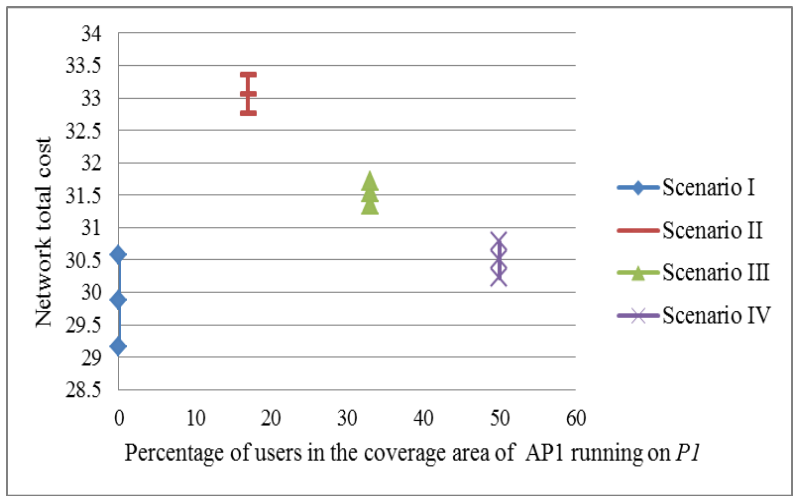

Figure 2. The $95 \%$ CI of the network total cost for case $1(\alpha=\beta=1)$.

Figure 2 shows the average network total cost for the different considered scenarios. We see that this cost has the lowest value when there is no user in the coverage of AP1, this is because this AP is in sleep mode in $100 \%$ of the snapshots . Moreover the network total cost decreases when the percentage of users in the AP1 increases. This is because when users are concentrated in one AP the transmission delay will increase whereas when they are distributed between the two cells this delay will decrease.

For the second case where $\alpha=0.01$ and $\beta=0.99$, the delay cost of the network total cost is highlighted compared to the power cost. Table V(a) shows that even when there is no users in the cell, the corresponding AP runs on $P 2$ for $95 \%$ of the snapshots. For the three scenarios (Table V(b)), the two APs run on $P 2$. Note that in this case, the problem minimizes the delay regardless of the power saving. Thus, putting the APs on $P 2$ will lead to an increase in users throughput and therefore will minimize the delay cost. 
(a) Scenario I

\begin{tabular}{c|c|c|c} 
& $P 1$ & $P 2$ & $S$ \\
\hline AP1 & 0 & 0 & $100 \%$ \\
\hline AP2 & 0 & $100 \%$ & 0
\end{tabular}

(c) Scenario III

\begin{tabular}{c|c|c|c} 
& $P 1$ & $P 2$ & $S$ \\
\hline AP1 & $29 \%$ & $34 \%$ & $37 \%$ \\
\hline AP2 & $17 \%$ & $69 \%$ & $14 \%$
\end{tabular}

\begin{tabular}{c|c|c|c} 
& $P 1$ & $P 2$ & $S$ \\
\hline AP1 & $22 \%$ & $7 \%$ & $71 \%$ \\
\hline AP2 & $2 \%$ & $93 \%$ & $5 \%$
\end{tabular}

(d) Scenario IV

\begin{tabular}{c|c|c|c} 
& $P 1$ & $P 2$ & $S$ \\
\hline AP1 & $26 \%$ & $49 \%$ & $25 \%$ \\
\hline AP2 & $23 \%$ & $63 \%$ & $14 \%$
\end{tabular}

Table VI

THE APS STATE FOR THE DIFFERENT SCENARIOS FOR CASE $3(\alpha=0.99$ AND $\beta=0.01$ ).

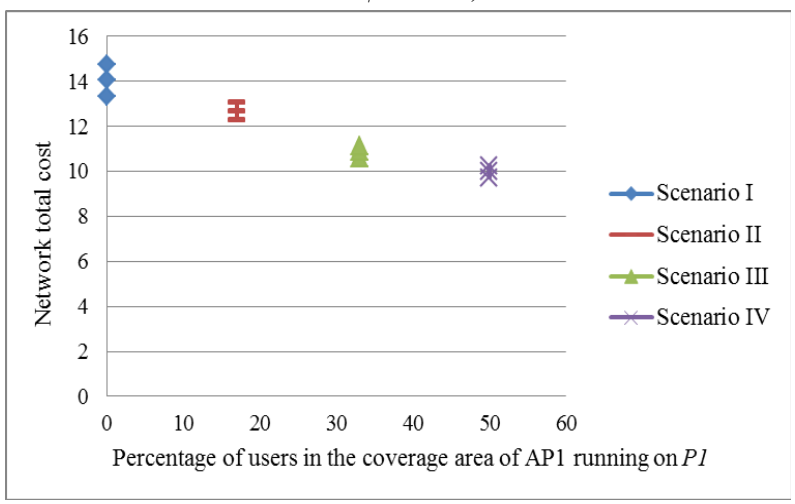

Figure 3. The $95 \% \mathrm{CI}$ of the network total cost for case $2(\alpha=0.01$ and $\beta$ $=0.99)$.

In Figure 3 the average network total cost decreases as the percentage of users in the first AP increases. As $\beta=0.99$, the network total cost here reflects the delay cost. Moreover, the power cost is constant since the two AP always run on $P 2$ in all scenarios. Therefore the delay decreases when the number of users is distributed between the cells.

In the third case where $\alpha=0.99$ and $\beta=0.01$, we aim to save power. For scenario I, we see in Table VI(a) that AP1 is always in sleep mode while AP2 runs on $P 2$. In fact, putting AP2 on $P 2$ increases the users throughput. For scenario II (Table VI(b)), AP1 is in sleep mode for $71 \%$ of the snapshots and AP2 runs on $P 2$ for $93 \%$ of the snapshots. From this scenario, we can conclude that when the percentage of users in a given cell is low, for example $17 \%$, we can put the corresponding AP into sleep mode and keep the other one running on $P 2$. For the two remaining scenarios (Tables VI(c) and VI(d)), the AP1 state is almost evenly distributed among the three states and the second one runs on $P 2$ for more than $60 \%$ of the snapshots. In this case the decision on which state to run the APs becomes more difficult, hence we provide in the following the results for the state couples of the APs (AP1 state, AP2 state) for the different snapshots. For the scenario III, we see in Figure 4(a) that the state where AP1 is in sleep mode and AP2 runs on $P 2$, denoted by the couple $(S, P 2)$, has the highest percentage at $37 \%$, followed by the couple $(P 1, P 2)$ at $24 \%$. Thereupon, for a similar users distribution in the cells, we can choose to put AP2 on $P 2$, and to put AP1 into sleep mode or on $P 1$ : this decision depends on how many users are close to which AP. For the scenario where users are evenly distributed between the two cells (Figure 4(b)), the percentage of the couples $(S, P 2),(P 1, P 2)$ are almost close and equal to

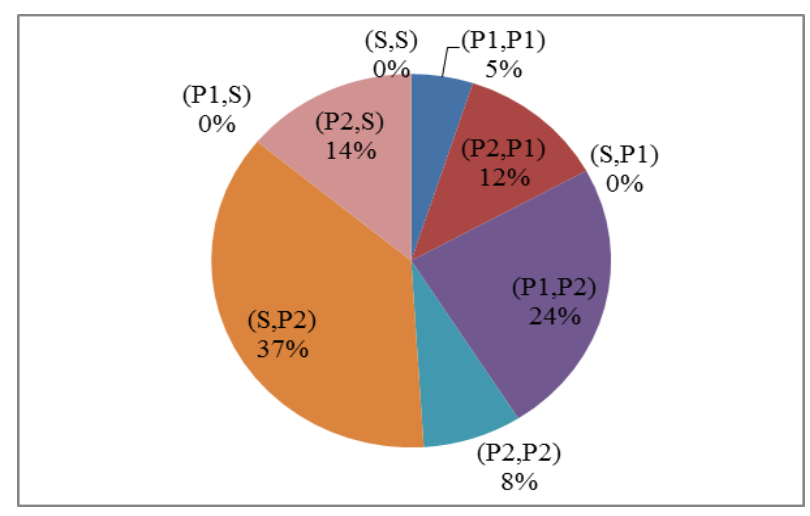

(a) Scenario III

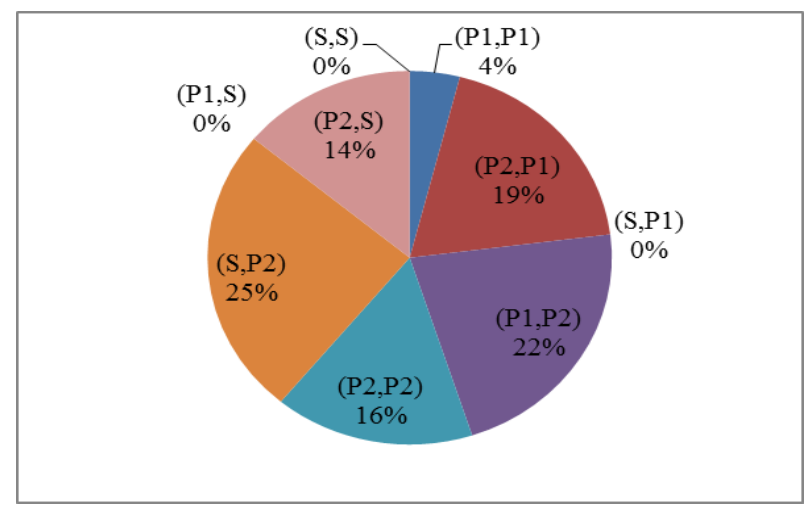

(b) Scenario IV

Figure 4. The percentage of the couple (AP1 state, AP2 state) for case $3(\alpha$ $=0.99$ and $\beta=0.01)$ (a) (b).

$25 \%$ and $22 \%$ respectively. The couple $(P 2, P 2)$ has relatively in this scenario a high percentage with respect to the other scenarios in this case.

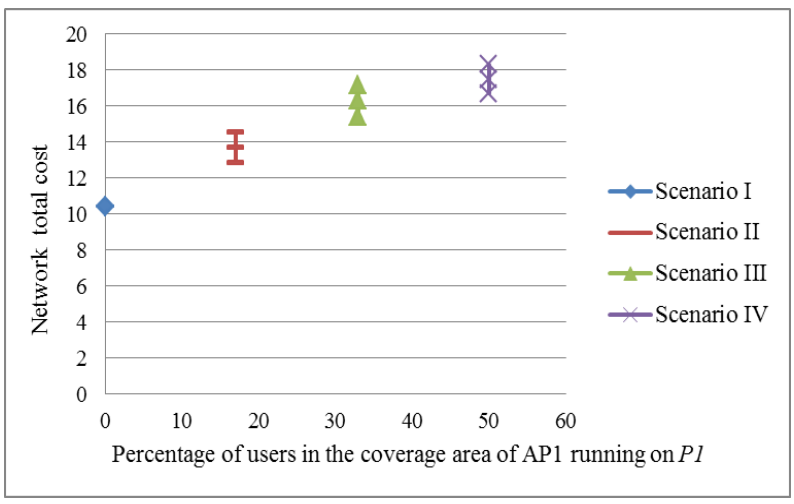

Figure 5. The $95 \% \mathrm{CI}$ of the network total cost for case $3(\alpha=0.99$ and $\beta$ $=0.01$ ).

The average network total cost in this case reflects the power cost. In Figure 5 we see that the network total cost increases as the percentage of users in the first AP increases. When the percentage of users in the first AP increases, this AP switches its mode from sleep to first and second power level causing the power cost to increase. 
In this paper, we provide basic simulation context with small network instances to give insights into the structure of the optimal solution. We choose small values of $N_{a p}$, $N_{l}$ and $N_{u}$ because the time complexity of the exhaustive search algorithm depends on these parameters as explained in Section III. For a small number of APs compared to the number of users in the network, the APs tend to run on the highest power level and users will experience bad QoS in terms of delay. On the opposite side, for a small number of users compared to the larger number of APs in the network, more APs tend to switch to sleep mode or run on the lowest power level and users will experience good QoS. Furthermore, we choose for simplicity $N_{l}=2$, if we increase $N_{l}$, the granularity will be finer but the solution will be more complex. In practice, the transmission power of an AP is discrete and the maximum number of the transmit power level is equal to 5 or 6 depending on the used standard (i.e, IEEE $802.11 \mathrm{a}, \mathrm{b}, \mathrm{g}$ or $\mathrm{n}$ ) and on the AP manufacturer (i.e, Cisco, Aruba, ...).

\section{CONClusion}

In this paper, we developed a theoretical multiobjective framework for the problem of power saving and satisfying user $\mathrm{QoS}$ in green wireless access network. We specifically introduced an optimization approach that simultaneously minimizes the network power consumption and the sum of data unit delays of all users in the network. The optimal solution of the problem is obtained by using an exhaustive search algorithm. Due to the high computational complexity of this algorithm in large scenarios, simulation were performed in a small network to give insight into the optimal solution. Precisely, different cases reflecting various decision preferences were studied by tuning the weights of the power and delay components of the network total cost. Regarding these preferences and under various spatial users distribution, numerical results show that our solution selects the optimal network configuration by putting some APs to sleep mode, adjusting the transmit power of others and associating user devices to the corresponding APs. Moreover, we considered the WLAN as an example of wireless access network. In fact, the fair rate model is a characteristic of the IEEE 802.11 technology [13], and our optimization model can be easily adapted to other wireless technologies (such as WiMAX, LTE, 2G/3G). For future work, we need to examine heuristic methods to solve the problem for larger scenarios.

\section{REFERENCES}

[1] F. Moety, M. Ibrahim, S. Lahoud, and K. Khawam, Distributed Heuristic Algorithms for RAT Selection in Wireless Heterogeneous Networks, in proceeding of Wireless Communications and Networking Conference (IEEE WCNC), France, 2012.

[2] K. Khawam, M. Ibrahim, J. Cohen, S. Lahoud, and S. Tohme, Individual vs. Global Radio Resource Management in a Hybrid Broadband Network, in proceeding of International Conference on Communications (IEEE ICC), Japan, 2011.

[3] H. Toure, ICTs and Climate Change-the ITU perspective, Climate Action 2008, www.climateactionprogramme.org.

[4] F. Richter, A. J. Fehske, G. P. Fettweis, Energy Efficiency Aspects of Base Station Deployment Strategies for Cellular Networks, in proceeding of Vehicular Technology Conference (VTC) Fall, USA, 2009.
[5] S. Dimatteo, P. Hui, B. Han and V. Li, Cellular traffic offloading through WiFi networks, in proceeding of 8th IEEE International Conference on Mobile Ad-hoc and Sensor Systems (IEEE MASS), Spain, 2011.

[6] B. Badic, T. O'Farrrell, P. Loskot, J. He, Energy Efficient Radio Access Architectures for Green Radio: Large versus Small Cell Size Deployment, in proceeding of Vehicular Technology Conference (VTC) Fall, USA, 2009.

[7] O. Eunsung, B. Krishnamachari, Energy Savings through Dynamic Base Station Switching in Cellular Wireless Access Networks, in proceeding of GLOBECOM, USA, 2010.

[8] A. J. Fehske, F. Richter, and G. P. Fettweis, Energy efficiency improvements through micro sites in cellular mobile radio networks, in proceeding of Workshop on Green Communication in conjunction with IEEE GLOBECOM, USA, 2009.

[9] W. Wang, and G. Shen, Energy Efficiency of Heterogeneous Cellular Network, in proceeding of 72nd Vehicular Technology Conference (VTC) Fall, Canada, 2010.

[10] S. Tombaz, M. Usman, and J. Zander, Energy Efficiency Improvements Through Heterogeneous Networks in Diverse Traffic Distribution Scenarios, in proceeding of 6th International ICST Conference on Communications and Networking in China (Chinacom), China, 2011.

[11] J. Lorincz, A. Capone, and M. Bogarelli, Energy savings in wireless access networks through optimized network management, in proceeding of 5th International Symposium on Wireless Pervasive Computing (ISWPC), Italy, 2010.

[12] K. Son, H. Kim, Y. Yi, and B. Krishnamachari, Base Station Operation and User Association Mechanisms for Energy-Delay Tradeoffs in Green Cellular Networks, IEEE Journal on Selected Areas in Communications, vol. 29, no. 8, 2011.

[13] M. Heusse, F. Rousseau, G. Berger-Sabbatel, and A. Duda, Performance Anomaly of $802.11 \mathrm{~b}$, in proceeding of INFOCOM, USA, 2003. 